\title{
Oxidative and Antioxidative Stress Markers in Relation to Left Ventricular Dysfunction and Gender
}

Indrani Garre ${ }^{1}$ Raju Nallagasu ${ }^{2}$ Malleshwar Rao Dangati ${ }^{1} \quad$ Indumathi Bobbala ${ }^{3} \quad$ Ravikiran Muddada $^{1}$

Address for correspondence Indrani Garre, PhD Student, Department of Cardiology, Nizam's Institute of Medical Sciences (NIMS), Punjagutta 500082, Hyderabad, Telangana, India (e-mail: indrani.garre@gmail.com).

Abstract
Keywords
- malondialdehyde
- glutathione
- nitric oxide
- oxidative stress
markers
- antioxidative stress
markers
- left ventricular
dysfunction
- percutaneous
coronary intervention
- gender

Objective The aim of this study was to investigate the relationship between oxidative and antioxidative stress markers with presence of left ventricular dysfunction (LVD) and the severity of LVD with coronary artery disease (CAD) in patients who have undergone percutaneous coronary intervention $(\mathrm{PCI})$. Further, the role of oxidative and antioxidative stress markers on gender was also investigated.

Methods This was an observational prospective pilot study of patients diagnosed with CAD with LVD who underwent PCI at the center from June 2017 to December 2017. Based on the ejection function (EF), patients were categorized into three groups: mild $(>40-50 \%)$, moderate $(>35-40 \%)$, and severe $(\leq 35 \%)$. The oxidative and antioxidative stress markers (malondialdehyde [MDA], glutathione [GSH], and nitric oxide [NO]) were studied and compared in these groups and in both genders as subanalysis.

Results Total 33 patients were enrolled, of whom 23 were male (69.7\%) and 10 were female (30.3\%). Mean age of the study population was $58.8 \pm 9.3$ years. Significant elevation of MDA and NO was seen in 33 (100\%) and 26 (78.8\%), respectively, and decreased GSH was seen in 30 (90.9\%). There was no significant difference with respect to oxidative and antioxidative stress markers and severity of LVD (MDA, $p=0.25$; NO, $p=0.79$; and GSH, $p=0.2$ ) despite elevated MDA levels in all patients. The subanalysis was done to see the gender effect with oxidative and antioxidative stress markers (MDA, $p=0.29$; NO, $p=0.10$; and $\mathrm{CSH}, p=0.50$ ), and they all were insignificant.

Conclusion In this study, there was no significant relationship of oxidative and antioxidative stress markers on the degree of LVD even though the elevated MDA levels suggestive of increased oxidative stress were seen in all patients. The further analysis of gender in relation to oxidative and antioxidative stress markers was also insignificant.

\section{Introduction}

The oxidative stress (OS) state is characterized by an increase in the amount of reactive oxygen and nitrogen species that overwhelm the antioxidant defense systems of the body. This state is implicated in the pathophysiology of multiple chronic conditions, including cardiovascular disease (CVD). ${ }^{1}$ Inflammation and oxidation play a critical role in the pathophysiology of atherosclerosis leading to CVD., ${ }^{2,3}$ Reactive oxygen species (ROS) initiate cellular tissue damage by modifying lipids, proteins, and DNA. Oxidative modification of LDL by free radicals results in negative effects on vascular function such as reduction in nitric oxide (NO) levels, endothelial apoptosis, increase in smooth muscle cell proliferation, and synthesis of proinflammatory molecules. ${ }^{4} \mathrm{NO}$ is a vasodilator agent derived from the endothelium, which also 
has an inhibitory role in thrombocyte aggregation, leukocyte adhesion, smooth muscle cell proliferation, and low-density lipoprotein (LDL) oxidation. The decrease in synthesis and bioavailability of NO is an important step in the development of atherosclerosis. ${ }^{5}$

Malondialdehyde (MDA), a carbonyl group produced during lipid peroxidation, is used widely in determining OS. Studies have demonstrated that MDA is an important marker of lipid peroxidation and that progression of atherosclerosis is correlated with OS and can be followed up using MDA. ${ }^{6}$ Glutathione (GSH), a sulfhydryl ( $\mathrm{SH}$ )-containing tripeptide, plays a role in detoxifying oxygen radicals and thus may prevent cellular damage from OS. GSH deficiency contributes to $\mathrm{OS}$, which plays a key role in aging and the pathogenesis of many chronic diseases.

In vitro and in vivo research and experiments in animal models have demonstrated that excess free-radical generation or impaired antioxidant function adversely affects the cardiac myocyte function and contributes to congestive heart failure (CHF). However, whether free-radical processes have a pathophysiologic role in the vast majority of patients with CHF due to ischemia is unclear. Theoretically, the authors may expect more OS as the severity of left ventricular dysfunction (LVD) increases. Many studies have described the correlation of risk factors (hypertension [HTN], diabetes mellitus [DM], smoking, family history [FH]) and type of CAD with oxidative and antioxidative stress markers. Morbidity and mortality following CAD are more in women than in men even after revascularization despite nullifying the gender bias in the treatment. ${ }^{7}$ The role of OS contributing to this increased risk has never been evaluated. This study mainly concentrated to see the relation between severity of LVD and gender with oxidative and antioxidative stress markers.

\section{Material and Methods}

This was an observational prospective pilot study. Patients diagnosed with CAD with LVD (left ventricular ejection fraction $[\mathrm{LVEF}]<50 \%$ ) who underwent percutaneous coronary intervention $(\mathrm{PCI})$ were included in this study from June 2017 to December 2017. Patients with end-stage renal disease (estimated glomerular filtration rate $[$ GGFR] $<30 \%$ ) were excluded. Clinical, echocardiographic, and angiographic details were noted. Based on the ejection function (EF) obtained on two-dimensional (2D) echocardiography, the patients were categorized into three groups: $\operatorname{mild}(>40-50 \%)$, moderate $(>35-40 \%)$, and severe $(\leq 35 \%)$. The oxidative and antioxidative stress markers (MDA, GSH, and NO) were obtained in all patients, and a comparison between the three groups was done. Comparison between both genders was also done.

\section{Collection of Samples}

Ten-milliliter venous blood was collected from every patient to 200 units heparin containing sterile vials. The blood was centrifuged immediately at 3,000 rpm (revolution per minute) for 15 minutes. The plasma was separated and kept on $-80^{\circ} \mathrm{C}$ until the time of analysis.

\section{Estimation of Malondialdehyde}

Malondialdehyde is an auxiliary result of lipid peroxidation and it was evaluated utilizing the colorimetric response of thiobarbituric corrosive (TBA) (BDH Fine Chemicals) in the plasma tests. Since the measure assesses the measure of TBA-responsive substance, for instance MDA, it is otherwise called TBARS (TBA-receptive substance) test and it gives a record of the degree of advance of lipid peroxidation.

To $0.5 \mathrm{~mL}$ of the serum, $0.5 \mathrm{~mL}$ of $30 \%$ trichloroacetic corrosive (TCA) (BDH Fine Chemicals) and $100 \mu \mathrm{L}$ of $1 \%$ TBA reagent were included and secured with aluminum thwart. The tubes were kept for 1 hour at $80^{\circ} \mathrm{C}$ in a shaking water bath. Then the tubes were transferred for 10 minutes to ice-cold water. The tubes were centrifuged at 3,000 rpm for 15 minutes. The supernatants were collected. The absorbance of the supernatant was checked at $540 \mathrm{~nm}$ against $1 \mathrm{~mL}$ of distilled water as blank at room temperature. The normal range is 2.70 to 4.50 nanomole $/ \mathrm{mL}^{8}$

\section{Estimation of Nitric Oxide}

To $1.0 \mathrm{~mL}$ of serum in radioimmunoassay (RIA) tube, add $40 \mu \mathrm{L}$ of $1 \mathrm{~N} \mathrm{NaOH}$ and $400 \mu \mathrm{L}$ of $120 \mathrm{mM}$ zinc sulfate $\left(\mathrm{ZnSO}_{4}\right)$ and Vortex for 30 seconds and is then centrifuged at 3,000 rpm for 10 minutes. Take $0.5 \mathrm{~mL}$ of supernatant into a clean, dry glass tube, and add $0.5 \mathrm{~mL}$ of vanadium (III) chloride (VCL3), $0.25 \mathrm{~mL} 2 \%$ sulfanilamide (SULF), and $0.25 \mathrm{~mL} 0.1 \%$ $\mathrm{N}$-(1-Naphthyl) ethylenediamine dihydrochloride (NEDD). Vortex for 15 seconds is incubated at $37^{\circ} \mathrm{C}$ for 30 minutes in dark. Read at $540 \mathrm{~nm}$ against a blank ( $1 \mathrm{~mL}$ of distilled water). Normal range is 24.87 to $48.23 \mu \mathrm{M} / \mathrm{L} .{ }^{9.10}$

\section{Estimation of Glutathione}

To $80 \mu \mathrm{L}$ of serum in RIA tube add $300 \mu \mathrm{L}$ of diluted buffer and $100 \mu \mathrm{L}$ of 5,5'-dithiobis (2-nitrobenzoic acid) (DTNB) and $1.6 \mathrm{~mL}$ of acetonitrile then incubate for 5 minutes at $37^{\circ} \mathrm{C}$. Centrifuge at $3,000 \mathrm{rpm}$ for 5 minutes. Measure the absorbance of the supernatant at $412 \mathrm{~nm}$ against blank $(1 \mathrm{~mL}$ of distilled water). Normal range is 509 to $657 \mu \mathrm{mol} / \mathrm{L}^{11,12}$

\section{Statistical Analysis}

All data were presented as the mean \pm standard deviation (SD). The groups were compared using one-way analysis of variance (ANOVA) for multiple comparisons between groups and two-tailed Student's $t$-test for comparison between the groups. Statistical analysis was done with Minitab version 17 by Minitab, Ltd. The $p$ values $<0.05$ were considered statistically significant.

\section{Results}

This was an observational prospective pilot study that enrolled 33 consecutive patients with mean age of $58.82 \pm 9.31$ years. 
Patients were diagnosed with coronary artery disease (CAD) with LVD who underwent PCI. Males were 23 (69.7\%) and 10 (30.3\%) were females. Twenty-six (78.8\%) patients presented with acute coronary syndrome (ACS). Nineteen (57.6\%) patients had severe LVD, 8 (24.2\%) had moderate LVD, and $6(18.2 \%)$ had mild LVD. Descriptive statistics and echo parameters are given in $\boldsymbol{- T a b l e ~} \mathbf{1}$.

In - Table 2 laboratory parameters along with OS markers are mentioned. Study population showed that mean $\mathrm{N}$-terminal prohormone of brain natriuretic peptide (NT-proBNP) and high-sensitivity C-reactive protein (hs-CRP) were elevated with relative leukocytosis.

All the patients had elevated MDA levels. GSH was shown normal in three $(9.1 \%)$ patients. NO was normal in seven (21.21\%) patients. Details are described in - Table 3.

Table 1 Descriptive and echo parameters of the whole study population

\begin{tabular}{|l|l|}
\hline Variable & Mean \pm SD \\
\hline Age (y) & $58.82 \pm 9.31$ \\
\hline Male (\%):Female (\%) & $23(69.7 \%): 10(30.3 \%)$ \\
\hline HTN (\%) & $20(60.6 \%)$ \\
\hline DM (\%) & $16(48.4 \%)$ \\
\hline ACS (\%):CSA (\%) & $26(78.3 \%): 7(21.2 \%)$ \\
\hline EDV (mL) & $134.61 \pm 24.17$ \\
\hline ESV (mL) & $86.52 \pm 22.28$ \\
\hline EDD (mm) & $5.22 \pm 0.79$ \\
\hline ESD (mm) & $4.05 \pm 0.88$ \\
\hline FS (\%) & $18.76 \pm 4.09$ \\
\hline EF (\%) & $37.15 \pm 8.11$ \\
\hline E/A ratio & $1.34 \pm 0.57$ \\
\hline DT (ms) & $130.1 \pm 61.4$ \\
\hline E/e ratio & $12.87 \pm 4.14$ \\
\hline
\end{tabular}

Abbreviations: ACS, acute coronary syndrome; CSA, chronic stable angina; DM, diabetes mellitus; DT, deceleration time; EDD, end-diastolic dimension; EDV, end-diastolic volume; EDV, end-systolic volume; EF, ejection fraction; ESD, end-systolic dimension; FS, fractional shortening; HTN, hypertension; SD, standard deviation.
The authors tested the significance of severity of LVD with oxidative and antioxidative markers, using one-way ANOVA. Details are mentioned in - Table $\mathbf{4}$, and box plots are shown in - Fig. 1.

Similarly, the authors also tested the correlation of oxidative and antioxidative markers with the type of CAD, and details are mentioned in - Table 5 .

Further subanalysis was done to test whether there was a correlation between the oxidative and antioxidative

Table 2 Details of laboratory parameters

\begin{tabular}{|l|l|}
\hline Laboratory parameters & Value \\
\hline Peak CPK $(\mathrm{U} / \mathrm{L})$ & $434 \pm 593$ \\
\hline hs-CRP $(\mathrm{mg} / \mathrm{L})$ & $56.6 \pm 57.3$ \\
\hline NT-proBNP $(\mathrm{pg} / \mathrm{mL})$ & $8,599 \pm 10,627$ \\
\hline Total leucocyte count (cells/L) & $16,266 \pm 32,831$ \\
\hline Hb $(\mathrm{gm} \%)$ & $12.43 \pm 2.11$ \\
\hline Serum creatinine $(\mathrm{mg} / \mathrm{dL})$ & $1.43 \pm 0.78$ \\
\hline GSH ( $\mu \mathrm{mol} / \mathrm{L})$ & $366.9 \pm 213.8$ \\
\hline MDA $(\mu \mathrm{mol} / \mathrm{L})$ & $9.75 \pm 1.14$ \\
\hline NO $(\mu \mathrm{mol} / \mathrm{L})$ & $22.73 \pm 26.66$ \\
\hline Total cholesterol $(\mathrm{mg} / \mathrm{dL})$ & $120.40 \pm 15.37$ \\
\hline HDL $(\mathrm{mg} / \mathrm{dL})$ & $48.20 \pm 13.31$ \\
\hline LDL $(\mathrm{mg} / \mathrm{dL})$ & $45.00 \pm 15.57$ \\
\hline Triglycerides $(\mathrm{mg} / \mathrm{dL})$ & $123.33 \pm 10.41$ \\
\hline LDH $(\mathrm{IU} / \mathrm{L})$ & $468.0 \pm 313.8$ \\
\hline
\end{tabular}

Abbreviations: CPK, creatine phosphokinase; GSH, glutathione; Hb, hemoglobin; HDL, high-density lipoprotein; hs-CRP; high-sensitivity C-reactive protein; $L D H$, lactate dehydrogenase; LDL, low-density lipoprotein; MDA, malondialdehyde; NO, nitric oxide; NT-proBNP, $\mathrm{N}$-terminal prohormone of brain natriuretic peptide.

Table 3 Oxidative and antioxidative stress markers details in this population

\begin{tabular}{|l|l|l|}
\hline Parameter & Normal & Abnormal \\
\hline Malondialdehyde (MDA) & 0 & $33(100 \%)$ \\
\hline Glutathione (GSH) & $3(9.1 \%)$ & $30(90.9 \%)$ \\
\hline Nitric oxide (NO) & $7(21.21 \%)$ & $26(78.79 \%)$ \\
\hline
\end{tabular}

Table 4 One-way ANOVA analysis based on LVD

\begin{tabular}{|c|c|c|c|c|c|}
\hline \multirow[t]{2}{*}{ Parameter } & \multicolumn{3}{|c|}{ LVD } & \multirow[t]{2}{*}{ F value } & \multirow[t]{2}{*}{$p$ Value } \\
\hline & Mild (mean \pm SD) & $\begin{array}{l}\text { Moderate (mean } \\
\quad \pm S D \text { ) }\end{array}$ & Severe (mean \pm SD) & & \\
\hline $\mathrm{GSH}(\mu \mathrm{mol} / \mathrm{L})$ & $323.9 \pm 46$ & $473 \pm 406$ & $335.7 \pm 99$ & 1.34 & 0.27 \\
\hline MDA $(\mu \mathrm{mol} / \mathrm{L})$ & $9.09 \pm 0.21$ & $9.70 \pm 1.29$ & $9.98 \pm 1.02$ & 1.44 & 0.25 \\
\hline $\mathrm{NO}(\mu \mathrm{mol} / \mathrm{L})$ & $18.8 \pm 33.0$ & $19.08 \pm 17.14$ & $25.53 \pm 28.76$ & 0.23 & 0.79 \\
\hline
\end{tabular}

Abbreviations: ANOVA, analysis of variance; GSH, glutathione; LVD, left ventricular dysfunction; MDA, malondialdehyde; NO, nitric oxide; SD, standard deviation. 
markers with gender, and all were insignificant. The details are mentioned in - Table 6.

As there was no difference of these oxidative and antioxidative stress markers, the authors further checked whether there was any difference in other parameters between the

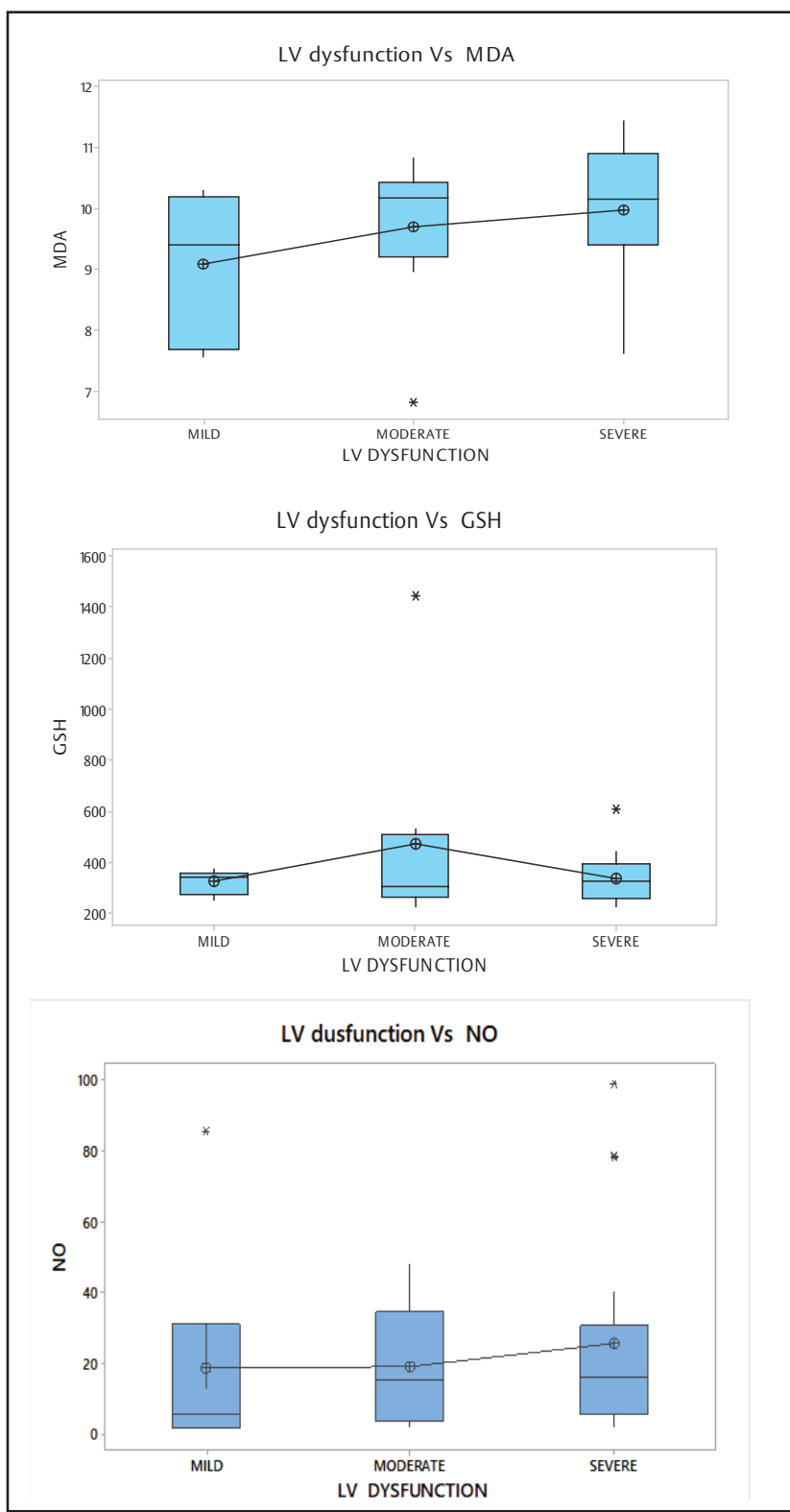

Fig. 1 Box plots of oxidative and antioxidative markers based on left ventricular (LV) dysfunction. GSH, glutathione; MDA, malondialdehyde; NO, nitric oxide. males and females. Details are mentioned in - Tables $\mathbf{7}$ and $\mathbf{8}$. Females were older and anemic with more cases of diabetes and hypertension. All other parameters including the severity of LVD, echo diameters, volumes, and other laboratory parameters such as renal and NT-proBNP levels were comparable. The authors have not seen the lipid abnormalities even though they are the main contributor to atherosclerosis as all the patients are already on medication.

\section{Discussion}

It is widely established that OS plays a pivotal role in the development and pathogenesis of CAD and its complications. ${ }^{13}$ Studies have demonstrated that OS may play different roles in stable CAD and ACS. Many studies have described the correlation of risk factors (HTN, DM, SM, FH) and type of CAD with oxidative and antioxidative stress markers. However, there is a paucity of data on these markers in LVD and gender differences. This pilot study mainly concentrated on the relation between LVD with oxidative and antioxidative stress markers, and further analysis was needed to see the gender effect on oxidative and antioxidative stress markers.

An increase in the levels of oxidized proteins and reduced antioxidant defenses (OS state) in essential hypertensive patients correlates with a worse systolic function and reduced diastolic function. ${ }^{14}$ Kameda et a ${ }^{15}$ provided clinical evidence for a link between OS, matrix metalloproteinase (MMP) activation, and LV dilatation in a study in 47 patients with ischemic heart disease (IHD) who underwent coronary artery bypass graft surgery. Even in this study oxidative proteins were increased in the study population suggested by elevated MDA in all patients.

Oxidative stress has long been involved in clinical and experimental CHF. The condition reflects an imbalance between the production of reactive gas species and endogenous antioxidant defense mechanisms. Elevated OS markers have been correlated with myocardial dysfunction and overall severity of heart failure. The authors hypothesize that the greater LVD would lead to the OS marker elevation and depletion of antioxidative markers. However, in this study, the authors observed that there was no significant relationship between the degree of LVD with oxidative and antioxidative stress markers even though all the patients had elevated MDA levels.

Overall, biological differences in atherosclerosis between men and women have been suggested but are not yet clarified, and they will need further research in the future. This

Table 5 One-way ANOVA analysis based on the type of CAD

\begin{tabular}{|l|l|l|l|l|}
\hline \multirow{2}{*}{ Parameter } & \multicolumn{4}{|c|}{ Type of CAD } \\
\cline { 2 - 5 } & ACS (mean \pm SD) & CSA (mean \pm SD) & F Value & $p$ Value \\
\hline GSH $(\mu \mathrm{mol} / \mathrm{L})$ & $378.2 \pm 238.1$ & $324.9 \pm 70.6$ & 0.34 & 0.56 \\
\hline $\mathrm{MDA}(\mu \mathrm{mol} / \mathrm{L})$ & $9.61 \pm 1.22$ & $10.27 \pm 0.56$ & 1.93 & 0.17 \\
\hline $\mathrm{NO}(\mu \mathrm{mol} / \mathrm{L})$ & $20.6 \pm 22.24$ & $30.6 \pm 40.5$ & 0.78 & 0.38 \\
\hline
\end{tabular}

Abbreviations: ACS, acute coronary syndrome; ANOVA, analysis of variance; CAD, coronary artery disease; CSA, chronic stable angina; $\mathrm{GSH}$, glutathione; MDA, malondialdehyde; NO, nitric oxide; SD, standard deviation. 
Table 6 Comparison of the oxidative and antioxidative markers based on gender

\begin{tabular}{|l|l|l|l|}
\hline Variable & Male $($ mean \pm SD) & Female (mean \pm SD) & $p$ Value \\
\hline GSH $(\mu \mathrm{mol} / \mathrm{L})$ & $380.2 \pm 243.4$ & $336.2 \pm 127.3$ & 0.50 \\
\hline $\mathrm{MDA}(\mu \mathrm{mol} / \mathrm{L})$ & $9.63 \pm 1.26$ & $10.02 \pm 0.79$ & 0.29 \\
\hline $\mathrm{NO}(\mu \mathrm{mol} / \mathrm{L})$ & $16.72 \pm 21.81$ & $36.6 \pm 32.6$ & 0.10 \\
\hline
\end{tabular}

Abbreviations: GSH, glutathione; MDA, malondialdehyde; NO, nitric oxide; SD, standard deviation.

Table 7 Comparison of demographics and laboratory parameters in males and females

\begin{tabular}{|c|c|c|c|}
\hline Variable & Male (mean \pm SD) & Female (mean \pm SD) & $p$ Value \\
\hline Number & 23 & 10 & \\
\hline Age $(y)$ & $56.17 \pm 9.32$ & $64.90 \pm 6.06$ & 0.004 \\
\hline $\mathrm{DM}(\%)$ & $9(39.1 \%)$ & $7(70 \%)$ & 0.08 \\
\hline HTN (\%) & $11(47.9 \%)$ & $9(90 \%)$ & 0.003 \\
\hline SM (\%) & $14(60 \%)$ & 0 & 0.000 \\
\hline Alcoholic (\%) & $9(39.1)$ & 0 & 0.000 \\
\hline Other risk factors (\%) & $6(26 \%)$ & $6(60 \%)$ & 0.05 \\
\hline Previous history of CAD & $3(13.04 \%)$ & $1(10 \%)$ & 0.79 \\
\hline $\mathrm{Hb}(\mathrm{gm} \%)$ & $13.139 \pm 1.970$ & $10.830 \pm 1.517$ & 0.001 \\
\hline Serum creatinine $(\mathrm{mg} / \mathrm{dL})$ & $1.322 \pm 0.784$ & $1.690 \pm 0.743$ & 0.21 \\
\hline Peak CPK (U/L) & $432 \pm 650$ & $440 \pm 466$ & 0.97 \\
\hline $\mathrm{LDH}(\mathrm{IU} / \mathrm{L})$ & $504.5 \pm 358.4$ & $384.0 \pm 157.6$ & 0.19 \\
\hline hs-CRP (mg/L) & $49.1 \pm 52.1$ & $79.1 \pm 74.3$ & 0.49 \\
\hline NT-proBNP (pg/mL) & $6,222 \pm 7,168$ & $12,674 \pm 14,626$ & 0.31 \\
\hline Total cholesterol (mg/dL) & $111.67 \pm 12.50$ & $134 \pm 5.57$ & 0.10 \\
\hline $\mathrm{HDL}(\mathrm{mg} / \mathrm{dL})$ & $43.33 \pm 14.47$ & $55.33 \pm 7.51$ & 0.29 \\
\hline $\mathrm{LDL}(\mathrm{mg} / \mathrm{dL})$ & $38 \pm 9.54$ & $50.33 \pm 17.04$ & 0.35 \\
\hline Triglycerides (mg/dL) & $125 \pm 14.1$ & $117.5 \pm 3.54$ & 0.60 \\
\hline $\mathrm{ST} 2$ (ng/mL) & $35.2 \pm 44.9$ & $86.7 \pm 113.0$ & 0.44 \\
\hline
\end{tabular}

Abbreviations: CAD, coronary artery disease; CPK, creatine phosphokinase; DM, diabetes mellitus; Hb, hemoglobin; HDL, high-density lipoprotein; hs-CRP; high-sensitivity C-reactive protein; HTN, hypertension; LDH, lactate dehydrogenase; LDL, low-density lipoprotein; NT-proBNP, N-terminal prohormone of brain natriuretic peptide; SD, standard deviation; SM, smoking.

Table 8 Comparison of echocardiographic parameters in males and females

\begin{tabular}{|l|l|l|l|}
\hline Variable & Male (Mean \pm SD) & Female (Mean \pm SD) & $p$ Value \\
\hline End-diastolic volume (EDV) (mL) & $134.78 \pm 25.72$ & $134.20 \pm 21.45$ & 0.95 \\
\hline End-systolic volume (ESV) (mL) & $87.35 \pm 22.96$ & $84.60 \pm 21.71$ & 0.75 \\
\hline End-diastolic dimension (EDD) (mm) & $5.33 \pm 0.86$ & $4.97 \pm 0.59$ & 0.18 \\
\hline End-systolic dimension (ESD) (mm) & $4.16 \pm 0.96$ & $3.79 \pm 0.63$ & 0.20 \\
\hline Fractional shortening (FS) (\%) & $18.57 \pm 4.49$ & $38.40 \pm 6.31$ & 0.65 \\
\hline Ejection fraction (EF) (\%) & $36.61 \pm 8.85$ & $1.25 \pm 0.58$ & 0.52 \\
\hline E/A ratio & $1.38 \pm 0.58$ & $123.6 \pm 45.9$ & 0.56 \\
\hline Declaration time (DT) (ms) & $132.9 \pm 67.8$ & $13.22 \pm 3.44$ & 0.65 \\
\hline E/e ratio & $12.72 \pm 4.47$ & 0.73 \\
\hline
\end{tabular}

is a pilot study to see whether OS is different in men and women attributing to the difference in atherosclerosis in both the sexes. However, this study showed no relationship between gender and oxidative and antioxidative markers.
Left ventricular dysfunction progresses to CHF and continues to contribute to high morbidity and mortality regardless of important advances in treatment. The use of new biomarkers for prognostication in LVD could be an impetus for the 
elucidation of CHF that could be therapeutically focused to enhance outcome in the affected person.

Even though MDA levels are elevated in all patients, there is no significant relationship between the degree of LVD and oxidative and antioxidative stress markers. It may indicate that the atherosclerotic plaque rupture has more impact than cell apoptosis. Whether OS contributes to differences in the pattern of atherosclerosis in both the sexes needs to be further studied in the larger population.

\section{Limitations}

This is an observational prospective pilot study. The results need to be confirmed with large population-based studies.

\section{Conclusion}

Malondialdehyde levels are elevated in all patients with CAD with LVD. However, there is no significant relationship between the degree of LVD and oxidative and antioxidative stress markers. The subanalysis of the study of gender did not have any correlation with oxidative and antioxidative stress markers.

\section{Acknowledgments}

Prof. M. Jyotsna, HOU-IV, and Dr. N. Lalita, Department of Cardiology, Nizam's Institute of Medical Sciences (NIMS), Hyderabad, for guidance, support, and encouragement.

Prof. K. Vijay Kumar, Department of CP\&T, NIMS, Hyderabad, for analysis of oxidative and antioxidative stress markers.

\section{References}

1 Harrison D, Griendling KK, Landmesser U, Hornig B, Drexler H. Role of oxidative stress in atherosclerosis. Am J Cardiol 2003;91(3A):7A-11A
2 Patel RS, Ghasemzadeh N, Eapen DJ, et al. Novel biomarker of oxidative stress is associated with risk of death in patients with coronary artery disease. Circulation 2016;133(4):361-369

3 Zhang J, Wang M, Li Z, et al. NADPH oxidase activation played a critical role in the oxidative stress process in stable coronary artery disease. Am J Transl Res 2016;8(12):5199-5210

4 Steinberg D. Low density lipoprotein oxidation and its pathobiological significance. J Biol Chem 1997;272(34):20963-20966

5 Furchgott RF, Zawadzki JV. The obligatory role of endothelial cells in the relaxation of arterial smooth muscle by acetylcholine. Nature 1980;288(5789):373-376

6 Draper HH, Hadley M. Malondialdehyde determination as index of lipid peroxidation. Methods Enzymol 1990;186:421-431

7 Shaw LJ, Bugiardini R, Merz CN. Women and ischemic heart disease: evolving knowledge. J Am Coll Cardiol 2009;54(17): 1561-1575

8 Vidyasagar J, Karunakar N, Reddy MS, Rajnarayana K, Surender T, Krishna DR. Oxidative stress and antioxidant status in acute organophosphorus insecticide poisoning. IJCP 2004;36(2):76-79

9 Miranda KM, Wink DA, Espey MG. A rapid, simple spectrophotometric method for simultaneous detection of nitrite and nitrate. Nitric Oxide 2001;5(1):62-71

10 Sastry KV, Moudgal RP, Mohan J, Tyagi JS, Rao GS. Spectrophotometric determination of serum nitrite and nitrate by copper-cadmium alloy. Anal Biochem 2002;306(1):79-82

11 Ellman GL. Tissue sulfhydryl groups. Arch Biochem Biophys 1959;82(1):70-77 (original determination)

12 Koster JF, Biemond P, Swaak AJ. Intracellular and extracellular sulphydryl levels in rheumatoid arthritis. Ann Rheum Dis 1986;45(1):44-46

13 Lin CP, Lin FY, Huang PH, et al. Endothelial progenitor cell dysfunction in cardiovascular diseases: role of reactive oxygen species and inflammation. BioMed Res Int 2013;2013:845037

14 Moreno-Ruíz LA, Ibarra-Quevedo D, Rodríguez-Martínez E, et.al. Oxidative stress state is associated with left ventricular mechanics changes, measured by speckle tracking in essential hypertensive patients: Oxidative Medicine and Cellular Longevity 2015. DOI: http://dx.doi.org/10.1155/2015/502107

15 Kameda K, Matsunaga T, Abe N, et al. Correlation of oxidative stress with activity of matrix metalloproteinase in patients with coronary artery disease. Possible role for left ventricular remodelling. Eur Heart J 2003;24(24):2180-2185 